Kragujevac Journal of Mathematics

Volume 45(4) (2021), Pages 499-519.

\title{
A SUBCLASS OF NOOR-TYPE HARMONIC $p$-VALENT FUNCTIONS BASED ON HYPERGEOMETRIC FUNCTIONS
}

\author{
HIBA F. AL-JANABY ${ }^{1}$ AND F. GHANIM ${ }^{2}$
}

\begin{abstract}
In this paper, we introduce a new generalized Noor-type operator of harmonic $p$-valent functions associated with the Fox-Wright generalized hypergeometric functions (FWGH-functions). Furthermore, we consider a new subclass of complex-valued harmonic multivalent functions based on this new operator. Several geometric properties for this subclass are also discussed.
\end{abstract}

\section{INTRODUCTION}

Harmonic function has fruitful applications not only in applied mathematics, but also in physics, engineering. It appears in differential equations, such as harmonic differential equations, wave equations, and heat equations. In geometric function theory (GFT), the famed authors Clunie and Sheil-Small [11] launched the study of harmonic univalent functions in 1984. In their investigates, they provided a class $\mathcal{S}_{\mathcal{H}}$ of harmonic functions $\varphi=\phi+\bar{\psi}$ that are univalent, sense-preserving which is $\left|\phi^{\prime}(z)\right|>\left|\psi^{\prime}(z)\right|$ in the unit disk $\mathbb{D}=\{z \in \mathbb{C}:|z|<1\}$, and normalized by the conditions $\varphi(0)=\varphi^{\prime}(0)-1=0$, where the regular(analytic) part $\phi$ and the co-regular part $\psi$ are defined as follows:

$$
\phi(z)=z+\sum_{\kappa=2}^{\infty} \mu_{\kappa} z^{\kappa}, \psi(z)=\sum_{\kappa=1}^{\infty} \nu_{\kappa} z^{\kappa}, \quad\left|\nu_{1}\right|<1 .
$$

In addition, they studied its geometric properties, which involves coefficient bounds, growth and distortion formulas. Note that, class $\mathcal{S}_{\mathcal{H}}$ reduces to the class $\mathcal{S}$ of regular univalent functions if the co-regular part $\psi$ is zero.

Key words and phrases. Harmonic multivalent function, convolution product, Noor integral operator, Fox-Wright generalized hypergeometric function.

2010 Mathematics Subject Classification. Primary: 30C45. Secondary: 11M35, 30C10.

DOI 10.46793/KgJMat2104.499J

Received: August 09, 2018.

Accepted: February 21, 2019. 
In 2001, Ahuja and Jahangiri [2] defined a more general class $\mathcal{S}_{\mathcal{H}(p)}$ of harmonic $p$-valent (multivalent) functions, $\varphi=\phi+\bar{\psi}$ that are sense-preserving in $\mathbb{D}$, and $\phi$ and $\psi$ are of the formula

$$
\phi(z)=z^{p}+\sum_{\kappa=p+1}^{\infty} \mu_{\kappa} z^{\kappa}, \psi(z)=\sum_{\kappa=p}^{\infty} \nu_{\kappa} z^{\kappa}, \quad\left|\nu_{p}\right|<1, p \in \mathbb{N}=\{1,2, \ldots\} .
$$

Note that, class $\mathcal{S}_{\mathcal{H}(p)}$ reduces to the class $\mathcal{M}_{p}$ of normalized regular $p$-valent functions if the co-regular part $\psi$ is zero. Consequently, the function $\varphi \in \mathcal{M}_{p}$ are expressed as:

$$
\varphi(z)=z^{p}+\sum_{\kappa=p+1}^{\infty} \mu_{\kappa} z^{\kappa} .
$$

Denoted by $\mathcal{N}_{\mathcal{H}(p)}$ the subclass of $\mathcal{S}_{\mathcal{H}(p)}$ consisting of functions $\varphi=\phi+\bar{\psi}$ such that the regular functions $\phi$ and $\psi$ are of the form

$$
\phi(z)=z^{p}-\sum_{\kappa=p+1}^{\infty}\left|\mu_{\kappa}\right| z^{\kappa}, \psi(z)=-\sum_{\kappa=p}^{\infty}\left|\nu_{\kappa}\right| z^{\kappa}, \quad\left|\nu_{p}\right|<1, p \in \mathbb{N}=\{1,2, \ldots\} .
$$

Convolution (Hadamard) product is a mathematical operation on two regular functions $\varphi_{1}$ and $\varphi_{2}$ to yield a third regular function $\varphi_{3}$. It is used to define various subclasses and linear operators in GFT. This concept owes its origin to Hadamard in 1899 [22]. In the harmonic functions case, Clunie and Sheil-Small [11] studied and defined the following convolution product: for any two functions $\varphi_{\imath} \in \mathcal{S}_{\mathcal{H}}$ of the form

$$
\varphi_{\imath}(z)=\phi_{\imath}(z)+\overline{\psi_{\imath}(z)}=z+\sum_{\kappa=2}^{\infty} \mu_{\kappa, \imath} z^{\kappa}+\overline{\sum_{\kappa=1}^{\infty} \nu_{\kappa, \imath} z^{\kappa}}
$$

where $\imath=1,2,\left|\nu_{1,1}\right|<1,\left|\nu_{1,2}\right|<1$, their convolution is denoted by $\varphi_{1} * \varphi_{2}$ and defined as

$$
\left(\varphi_{1} * \varphi_{2}\right)(z)=z+\sum_{\kappa=2}^{\infty} \mu_{\kappa, 1} \mu_{\kappa, 2} z^{\kappa}+\overline{\sum_{\kappa=1}^{\infty} \nu_{\kappa, 1} \nu_{\kappa, 2} z^{\kappa}}
$$

More generally, the convolution of two functions $\varphi_{\imath} \in \mathcal{S}_{\mathcal{H}(p)}$ is given by (see, [29]):

$$
\left(\varphi_{1} * \varphi_{2}\right)(z)=z^{p}+\sum_{\kappa=p+1}^{\infty} \mu_{\kappa, 1} \mu_{\kappa, 2} z^{\kappa}+\overline{\sum_{\kappa=p}^{\infty} \nu_{\kappa, 1} \nu_{\kappa, 2} z^{\kappa}}
$$

where

$$
\varphi_{\imath}(z)=\phi_{\imath}(z)+\overline{\psi_{\imath}(z)}=z^{p}+\sum_{\kappa=p+1}^{\infty} \mu_{\kappa, \imath} z^{\kappa}+\overline{\sum_{\kappa=p}^{\infty} \nu_{\kappa, \imath} z^{\kappa}}, \quad \imath=1,2,\left|\nu_{p, 1}\right|<1,\left|\nu_{p, 2}\right|<1 .
$$

Operators Theory has a significant role in the study GFT. Actually, operators are utilized in defining new subclasses. The technique of convolution has a remarkable part in the evolution of this area. Numerous differential and integral operators (linear operators) can be established in terms of the convolution. In 1915, Alexander [4] introduced the first integral operator on class $\mathcal{A}$ that includes normalized regular functions. Later, several well-known integral operators are investigated by complex 
analysts, such as Libera [26], Bernardi [9], Miller, Mocanu and Reade [27, 28], Pascu and Pescar [34], Ong et al. [33], Frasin [20], Frasin and Breaz [21], El-Ashwah, Aouf and El-Deeb [16], Deniz [13], Rahrovi [35], Al-Janaby and Ghanim [5], Al-Janaby, Ghanim, Darus [6], Al-Janaby [7] and others. The following are some important linear operators related to results in this study.

In 1975, Ruscheweyh [37] introduced the differential operator $D^{\tau} \varphi(z)$ so-called the Ruscheweyh differential operator as follows: for $\varphi \in \mathcal{A}, \tau>-1$ and $D^{\tau}: \mathcal{A} \rightarrow \mathcal{A}$ is given by

$$
D^{\tau} \varphi(z)=\frac{z}{(1-z)^{\tau+1}} * \varphi(z)=z+\sum_{\kappa=2}^{\infty} \frac{(\tau+1)_{\kappa-1}}{(\kappa-1) !} \mu_{\kappa} z^{\kappa}
$$

where $(a)_{\kappa}=\frac{\Gamma(a+\kappa)}{\Gamma(a)}$ denotes the Pochhammer symbol. Note that $D^{0} \varphi(z)=\varphi(z)$ and $D^{1} \varphi(z)=z \varphi^{\prime}(z)$.

Analogous manner to the Ruscheweyh operator, in 1999, the author Noor [31] presented an integral operator $I_{\tau} \varphi(z)$, namely Noor Integral of $\tau$-th order, as follows: for a function $\varphi \in \mathcal{A}$ and $\tau \in \mathbb{N}_{0}$, the Noor integral operator $I_{\tau}(z)$ is given by $I_{\tau}: \mathcal{A} \rightarrow \mathcal{A}$,

$$
I_{\tau} \omega(z)=\varphi_{\tau}^{(-1)}(z) * \varphi(z)=\left[\frac{z}{(1-z)^{\tau+1}}\right]^{-1} * \varphi(z)=z+\sum_{\kappa=2}^{\infty} \frac{\kappa !}{(\tau+1)_{\kappa-1}} \mu_{k} z^{\kappa}
$$

such that $\varphi_{\tau}(z) * \varphi_{\tau}^{(-1)}(z)=\frac{z}{(1-z)^{2}}$. Note that $I_{0} \varphi(z)=z \varphi^{\prime}(z), I_{1} \varphi(z)=\varphi(z)$. This version of integral operator is a considerable gadget in imposing several subclasses of regular functions.

On the other hand, special functions have been applied in GFT. In 1984, de Branges [12] employed hypergeometric function in proving the prominent problem called Bieberbach's conjecture. Since then, the study of hypergeometric function and its generalizations have attracted the attention of many function theorists. The important role played by special functions is defining new operators. The generalized hypergeometric function known as Fox-Wright generalized hypergeometric function (FWGH-function) is defined as: (see for example [19,40] and [41])

$$
\begin{aligned}
& \eta \mathcal{W}_{\delta}\left[\left(\rho_{l}, \mathcal{C}_{l}\right)_{1, \eta} ;\left(\sigma_{l}, \mathcal{D}_{l}\right)_{1, \delta} ; z\right]=\eta \mathcal{W}_{\delta}\left[\left(\rho_{1}, \mathcal{C}_{1}\right) \cdots\left(\rho_{\eta}, \mathfrak{C}_{\eta}\right) ;\left(\sigma_{1}, \mathcal{D}_{1}\right) \cdots\left(\sigma_{\delta}, \mathcal{D}_{\delta}\right) ; z\right] \\
= & \sum_{\kappa=0}^{\infty} \frac{\Gamma\left(\rho_{1}+\kappa \mathcal{C}_{1}\right) \Gamma\left(\rho_{2}+\kappa \mathcal{C}_{2}\right) \cdots \Gamma\left(\rho_{\eta}+\kappa \mathcal{C}_{\eta}\right)}{\Gamma\left(\sigma_{1}+\kappa \mathcal{D}_{1}\right) \Gamma\left(\sigma_{2}+\kappa \mathcal{D}_{2}\right) \cdots \Gamma\left(\sigma_{\delta}+\kappa \mathcal{D}_{\delta}\right)} \frac{z^{\kappa}}{\kappa !}
\end{aligned}
$$

$$
=\sum_{\kappa=0}^{\infty} \frac{\prod_{\jmath=1}^{\eta} \Gamma\left(\rho_{\jmath}+\kappa \mathcal{C}_{\jmath}\right)}{\prod_{\jmath=1}^{\delta} \Gamma\left(\sigma_{\jmath}+\kappa \mathcal{D}_{\jmath}\right)} \frac{z^{\kappa}}{\kappa !},
$$


where $\mathcal{C}_{\jmath}>0, \jmath=1,2, \ldots, \eta, \mathcal{B}_{\jmath}>0, \jmath=1,2, \ldots, \delta, 1+\sum_{\jmath=1}^{\eta} \mathcal{C}_{\jmath}-\sum_{\jmath=1}^{\delta} \mathcal{D}_{\jmath} \geq 0$, $\rho_{\jmath}+\kappa \mathfrak{C}_{\jmath} \neq 0,-1, \ldots, \jmath=1,2, \ldots, \eta, \kappa=0,1, \ldots, \sigma_{\jmath}+\kappa \mathcal{D}_{\jmath} \neq 0,-1, \ldots, \jmath=1,2, \ldots$, $\delta, \kappa=0,1, \ldots$ and $z \in \mathbb{C}$. The condition $1+\sum_{\jmath=1}^{\eta} \mathcal{C}_{\jmath}-\sum_{\jmath=1}^{\delta} \mathcal{D}_{\jmath} \geq 0$ is essential so that the series in (1.7) is absolutely convergent for all $z \in \mathbb{C}$, and is an entire function of $z$ (for details, see [25]). Special case of FWGH-function defined in (1.7), given as: if $\mathfrak{C}_{\jmath}=1, \jmath=1,2, \ldots, \eta, \mathcal{D}_{\jmath}=1, \jmath=1,2, \ldots, \delta, \eta \leq \delta+1$ and

$$
\Xi=\left(\prod_{\jmath=1}^{\delta} \Gamma\left(\sigma_{\jmath}\right)\right)\left(\prod_{\jmath=1}^{\eta} \Gamma\left(\rho_{\jmath}\right)\right)^{-1}
$$

then

$$
\Xi \eta \mathcal{W}_{\delta}\left[\left(\rho_{\jmath}, 1\right)_{1, \eta} ;\left(\sigma_{\jmath}, 1\right)_{1, \delta} ; z\right]=\eta \mathcal{F}_{\delta}\left[\left(\rho_{1}, \ldots \rho_{\eta} ; \sigma_{1}, \ldots \sigma_{\delta} ; z\right]\right.
$$

where $\eta \mathcal{F}_{\delta}\left[\left(\rho_{1}, \ldots, \rho_{\eta} ; \sigma_{1}, \ldots, \sigma_{\delta} ; z\right]\right.$ is a generalized hypergeometric function, [14]. Other special cases of FWGH-function were presented in [25].

In the well-known theory of regular univalent functions, there are numerous investigates on hypergeometric functions associated with classes of regular functions. In 2004, Ahuja and Silverman [1] discovered the corresponding connections between hypergeometric functions and harmonic univalent functions. Recently, the connections between WGHF and harmonic univalent functions were discussed by some authors, such that Murugusundaramoorthy and Raina [30], Sharma [39], Raina and Sharma [36], Ahuja and Sharma [3] and Hussain et al.[23]. In addition, several operators have been extended to harmonic functions by authors. For instance, Chandrashekar et al. [10], El-Ashwah, and Aouf [17] Yaşar and Yalçin [42], Seoudy [38], Al-Janaby [8] and others. Some previous studies that involving hypergeometric and FWGH functions are presented in this paper.

In 2004, Dziok and Raina [15] considered the linear operator $\left.W\left(\rho_{\jmath}, \mathcal{C}_{\jmath}\right)_{1, \eta} ;\left(\sigma_{\jmath}, \mathcal{D}_{\jmath}\right)_{1, \delta}\right]$ by means of FWGH-function on $\mathcal{A}$ as:

$$
\left.W\left(\rho_{\jmath}, \mathcal{C}_{\jmath}\right)_{1, \eta} ;\left(\sigma_{\jmath}, \mathcal{D}_{\jmath}\right)_{1, \delta}\right] \varphi(z)=z+\sum_{\kappa=2}^{\infty} \Xi \vartheta_{\kappa} \mu_{\kappa} z^{\kappa}
$$

where

$$
\vartheta_{\kappa}=\frac{\Gamma\left(\rho_{1}+(\kappa-1) \mathcal{C}_{1}\right) \Gamma\left(\rho_{2}+(\kappa-1) \mathcal{C}_{2}\right) \cdots \Gamma\left(\rho_{\eta}+(\kappa-1) \mathcal{C}_{\eta}\right)}{\Gamma\left(\sigma_{1}+(\kappa-1) \mathcal{D}_{1}\right) \Gamma\left(\sigma_{2}+(\kappa-1) \mathcal{D}_{2}\right) \cdots \Gamma\left(\sigma_{\delta}+(\kappa-1) \mathcal{D}_{\delta}\right)(\kappa-1) !},
$$

and $\Xi$ is defined in (1.8). Following that, in 2016, Hussain, Rasheed and Darus [23] introduced a new subclass of harmonic functions by using the extension of the above linear operator to harmonic functions. Also, they investigated various properties such as coefficient bounds, extreme points, and inclusion results and closed under an integral operator for this subclass. 
In 2006, the author Noor [32] again imposed the integral operator $I_{\tau}(\zeta, \xi ; \gamma)$ by employing the Gauss hypergeometric function as follows:

$$
I_{\tau}(\zeta, \xi ; \gamma) \varphi(z)=[z \mathcal{F}(\zeta, \xi ; \zeta ; z)]^{(-1)} * \varphi(z)=z+\sum_{\kappa=2}^{\infty} \frac{(\gamma)_{\kappa-1}(\tau+1)_{\kappa-1}}{(\zeta)_{\kappa-1}(\xi)_{\kappa-1}} \mu_{\kappa} z^{\kappa}
$$

where

$$
[z \mathcal{F}(\zeta, \xi ; \gamma ; z)] *[z \mathcal{F}(\zeta, \xi ; \gamma ; z)]^{(-1)}=\frac{z}{(1-z)^{\tau+1}}=z+\sum_{\kappa=2}^{\infty} \frac{(\tau+1)_{\kappa-1}}{(\kappa-1) !} z^{\kappa} .
$$

In 2008, Ibrahim and Darus [24] studied the following generalized integral operator $I_{\tau}\left[\left(\sigma_{\jmath}, \mathcal{D}_{\jmath}\right)_{1, \delta} ;\left(\rho_{\jmath}, \mathcal{C}_{\jmath}\right)_{1, \eta}\right]$ associated with FWGH-function on $\mathcal{A}$, where

$$
I_{\tau}\left[\left(\sigma_{\jmath}, \mathcal{D}_{\jmath}\right)_{1, \delta} ;\left(\rho_{\jmath}, \mathcal{C}_{\jmath}\right)_{1, \eta}\right] \varphi(z)=z+\sum_{\kappa=2}^{\infty} \frac{\prod_{\jmath=1}^{\delta} \Gamma\left(\sigma_{\jmath}+(\kappa-1) \mathcal{D}_{\jmath}\right)}{\prod_{\jmath=1}^{\eta} \Gamma\left(\rho_{\jmath}+(\kappa-1) \mathcal{C}_{\jmath}\right)}(\tau+1)_{\kappa-1} \mu_{\kappa} z^{\kappa}
$$

and

$$
\frac{\Gamma\left(\sigma_{1}\right) \cdots \Gamma\left(\sigma_{\delta}\right)}{\Gamma\left(\rho_{1}\right) \cdots \Gamma\left(\rho_{\eta}\right)}=1
$$

Posterior, in 2016, the authors El-Ashwah and Hassan [18] established the linear operator $\Theta_{\kappa}\left[\left(\rho_{\jmath}, \mathcal{C}_{\jmath}\right)_{1, \tau} ;\left(\nu_{\jmath}, \mathcal{D}_{\jmath}\right)_{1, \varsigma}\right]$ on the class $\mathcal{M}_{p}$ of regular $p$-valent functions in $\mathbb{D}$ as:

$$
\Theta_{\kappa}\left[\left(\rho_{\jmath}, \mathcal{C}_{\jmath}\right)_{1, \eta} ;\left(\sigma_{\jmath}, \mathcal{D}_{\jmath}\right)_{1, \delta}\right] \varphi(z)=z^{p}+\sum_{\kappa=p+1}^{\infty} \Xi \vartheta_{\kappa} \rho_{\kappa} z^{\kappa}
$$

where

$$
\vartheta_{\kappa}=\frac{\Gamma\left(\rho_{1}+(\kappa-p) \mathcal{C}_{1}\right) \Gamma\left(\rho_{2}+(\kappa-p) \mathcal{C}_{2}\right) \cdots \Gamma\left(\rho_{\eta}+(\kappa-p) \mathcal{C}_{\eta}\right)}{\Gamma\left(\sigma_{1}+(\kappa-p) \mathcal{D}_{1}\right) \Gamma\left(\sigma_{2}+(\kappa-p) \mathcal{D}_{2}\right) \cdots \Gamma\left(\sigma_{\delta}+(\kappa-p) \mathcal{D}_{\delta}\right)(\kappa-p) !},
$$

and $\Xi$ is defined in (1.8).

In this study, we continue our investigates in the theory of operators. Here we'll introduce a new generalized Noor-type operator of harmonic $p$-valent functions associated with FWGH-functions. We then define a new subclass and discuss several of its properties.

\section{Imposed Operator $\mathcal{J}_{p, \ell}^{\eta, \delta}\left[\sigma_{j} ; \rho_{\jmath}\right] \varphi(z)$}

This section proposes a new generalized Noor-type operator $\mathcal{J}_{p, \ell}^{\eta, \delta}\left[\sigma_{j} ; \rho_{j}\right] \varphi(z)$ for harmonic $p$-valent functions based on FWGH-function in (1.7).

By giving an extension of the FWGH-function in (1.7)

$$
\eta \mathcal{M}_{\delta}\left[\left(\rho_{\jmath}, \mathcal{C}_{\jmath}\right)_{1, \eta} ;\left(\sigma_{\jmath}, \mathcal{D}_{\jmath}\right)_{1, \delta} ; z\right]:=\Omega z^{p} \eta \mathcal{W}_{\delta}\left[\left(\rho_{\jmath}, \mathcal{C}_{\jmath}\right)_{1, \eta} ;\left(\sigma_{\jmath}, \mathcal{D}_{\jmath}\right)_{1, \delta} ; z\right]
$$




$$
=z^{p}+\sum_{\kappa=p+1}^{\infty} \frac{\Omega \prod_{\jmath=1}^{\eta} \Gamma\left(\rho_{\jmath}+(\kappa-p) \mathcal{C}_{\jmath}\right)}{\prod_{\jmath=1}^{\delta} \Gamma\left(\sigma_{\jmath}+(\kappa-p) \mathcal{D}_{\jmath}\right)} \cdot \frac{z^{\kappa}}{(\kappa-p) !}
$$

where

$$
\Omega=\left(\prod_{\jmath=1}^{\delta} \Gamma\left(\sigma_{\jmath}\right)\right)\left(\prod_{\jmath=1}^{\eta} \Gamma\left(\rho_{\jmath}\right)\right)^{-1} .
$$

We define a new generalization of the extended FWGH-function in (2.1) in terms of $\ell$-th convolution product as:

$$
\begin{aligned}
& \eta \mathcal{M}_{\delta}^{\ell}\left[\left(\rho_{\jmath}, \mathcal{C}_{\jmath}\right)_{1, \eta} ;\left(\sigma_{\jmath}, \mathcal{D}_{\jmath}\right)_{1, \delta} ; z\right] \\
:= & \underbrace{\eta \mathcal{M}_{\delta}\left[\left(\rho_{\jmath}, \mathcal{C}_{\jmath}\right)_{1, \eta} ;\left(\sigma_{\jmath}, \mathcal{D}_{\jmath}\right)_{1, \delta} ; z\right] * \cdots * \eta \mathcal{M}_{\delta}\left[\left(\rho_{\jmath}, \mathcal{C}_{\jmath}\right)_{1, \eta} ;\left(\sigma_{\jmath}, \mathcal{D}_{\jmath}\right)_{1, \delta} ; z\right]}_{\ell-\text { times }} \\
= & z^{p}+\sum_{\kappa=p+1}^{\infty}\left[\frac{\Omega \prod_{\jmath=1}^{\eta} \Gamma\left(\rho_{\jmath}+(\kappa-p) \mathcal{C}_{\jmath}\right)}{\prod_{\jmath=1}^{\delta} \Gamma\left(\sigma_{\jmath}+(\kappa-p) \mathcal{D}_{\jmath}\right)(\kappa-p) !}\right]^{\ell} z^{\kappa} .
\end{aligned}
$$

Then we introduce a new function $\left(\eta \mathcal{M}_{\delta}^{\ell}\left[\left(\rho_{\jmath}, \mathcal{C}_{\jmath}\right)_{1, \eta} ;\left(\sigma_{\jmath}, \mathcal{D}_{\jmath}\right)_{1, \delta} ; z\right]\right)^{-1}$ as:

$$
\begin{aligned}
& \left(\eta \mathcal{M}_{\delta}^{\ell}\left[\left(\rho_{\jmath}, \mathcal{C}_{\jmath}\right)_{1, \eta} ;\left(\sigma_{\jmath}, \mathcal{D}_{\jmath}\right)_{1, \delta} ; z\right]\right)^{-1} \\
= & z^{p}+\sum_{\kappa=p+1}^{\infty}\left[\frac{\prod_{\jmath=1}^{\delta} \Gamma\left(\sigma_{\jmath}+(\kappa-p) \mathcal{D}_{\jmath}\right)(\kappa-p) !}{\Omega \prod_{\jmath=1}^{\eta} \Gamma\left(\rho_{\jmath}+(\kappa-p) \mathcal{C}_{\jmath}\right)}\right]^{\ell} \frac{(\tau+p)_{\kappa-p}}{(\kappa-p) !} z^{\kappa},
\end{aligned}
$$

such that for $\tau>-p$

$$
\begin{aligned}
& \left(\eta \mathcal{M}_{\delta}^{\ell}\left[\left(\rho_{\jmath}, \mathcal{C}_{\jmath}\right)_{1, \eta} ;\left(\sigma_{\jmath}, \mathcal{D}_{\jmath}\right)_{1, \delta} ; z\right]\right) *\left(\eta \mathcal{M}_{\delta}^{\ell}\left[\left(\rho_{\jmath}, \mathcal{C}_{\jmath}\right)_{1, \eta} ;\left(\sigma_{\jmath}, \mathcal{D}_{\jmath}\right)_{1, \delta} ; z\right]\right)^{-1} \\
= & \frac{z^{p}}{(1-z)^{\tau+p}}=\sum_{\kappa=p}^{\infty} \frac{(\tau+p)_{\kappa-p}}{(\kappa-p) !} z^{\kappa} .
\end{aligned}
$$

Next, we consider the following linear operator: $\mathcal{J}_{p}^{\ell}\left[\left(\sigma_{\jmath}, \mathcal{D}_{\jmath}\right)_{1, \delta} ;\left(\rho_{\jmath}, \mathcal{C}_{\jmath}\right)_{1, \eta}\right]: \mathcal{M}_{p} \rightarrow \mathcal{M}_{p}$, where

$$
\begin{aligned}
& \mathcal{J}_{p}^{\ell}\left[\left(\sigma_{\jmath}, \mathcal{D}_{\jmath}\right)_{1, \delta} ;\left(\rho_{\jmath}, \mathcal{C}_{\jmath}\right)_{1, \eta}\right] \varphi(z)=\left(\eta \mathcal{M}_{\delta}^{\ell}\left[\left(\rho_{\jmath}, \mathcal{C}_{\jmath}\right)_{1, \eta} ;\left(\sigma_{\jmath}, \mathcal{D}_{\jmath}\right)_{1, \delta} ; z\right]\right)^{-1} * \varphi(z) \\
= & z^{p}+\sum_{\kappa=p+1}^{\infty}\left[\frac{\prod_{\jmath=1}^{\delta} \Gamma\left(\sigma_{\jmath}+(\kappa-p) \mathcal{D}_{\jmath}\right)(\kappa-p) !}{\Omega \prod_{\jmath=1}^{\eta} \Gamma\left(\rho_{\jmath}+(\kappa-p) \mathcal{C}_{\jmath}\right)}\right] \frac{(\tau+p)_{\kappa-p}}{(\kappa-p) !} \mu_{\kappa} z^{\kappa} .
\end{aligned}
$$


For brevity,

$$
\mathcal{J}_{p, \ell}^{\eta, \delta}\left[\sigma_{j} ; \rho_{\jmath}\right] \varphi(z)=\mathcal{J}_{p}^{\ell}\left[\left(\sigma_{\jmath}, \mathcal{D}_{\jmath}\right)_{1, \delta} ;\left(\rho_{\jmath}, \mathcal{C}_{\jmath}\right)_{1, \eta}\right] \varphi(z) .
$$

Remark 2.1. For suitably chosen parameters $p, \ell, \delta, \eta, \mathcal{C}_{1}, \mathcal{C}_{2}, \mathcal{D}_{1}, \rho_{1}, \rho_{2}$ and $\sigma_{1}$, the generalized Noor-type operator $\mathcal{J}_{p, \ell}^{\eta, \delta}\left[\sigma_{j} ; \rho_{\jmath}\right](2.6)$ reduces to some of the above linear operators. Thus, we obtain the following special cases.

- For $p=1, \ell=1, \delta=1, \eta=2, \mathcal{C}_{1}=\mathcal{C}_{2}=\mathcal{D}_{1}=1$, and $\rho_{1}=\rho_{2}=\sigma_{1}=1$ in (2.6), we gain the Ruscheweyh differential operator given by (1.5).

- For $p=1, \ell=1, \delta=1, \eta=2, \mathcal{C}_{1}=\mathcal{C}_{2}=\mathcal{D}_{1}=1, \rho_{1}=\rho_{2}=1+\tau$ and $\sigma_{1}=1$, the operator (2.6) provides the Noor integral operator in (1.6).

- By taking $p=1, \ell=1, \delta=1, \eta=2, \mathcal{C}_{1}=\mathcal{C}_{2}=\mathcal{D}_{1}=1, \rho_{1}=\zeta, \rho_{2}=\xi$ and $\sigma_{1}=\gamma$ in (2.6), gives us an integral operator defined by (1.9).

- If $p=1, \ell=1$ and $\Omega=1$, we yield the linear operator given by (1.10).

The generalized Noor-type operator $\mathcal{J}_{p, \ell}^{\eta, \delta}\left[\sigma_{j} ; \rho_{j}\right] \varphi(z)(2.6)$ when extended to harmonic $p$-valent function $\varphi=\phi+\bar{\psi}$ is defined by

$$
\mathcal{J}_{p, \ell}^{\eta, \delta}\left[\sigma_{j} ; \rho_{\jmath}\right] \varphi(z)=\mathcal{J}_{p, \ell}^{\eta, \delta}\left[\sigma_{j} ; \rho_{\jmath}\right] \phi(z)+\overline{\mathcal{J}_{p, \ell}^{\eta, \delta}\left[\sigma_{j} ; \rho_{\jmath}\right] \psi(z)},
$$

where

$$
\mathcal{\partial}_{p, \ell}^{\eta, \delta}\left[\sigma_{\jmath} ; \rho_{\jmath}\right] \phi(z)=z^{p}+\sum_{\kappa=p+1}^{\infty}\left[\frac{\prod_{\jmath=1}^{\delta} \Gamma\left(\sigma_{\jmath}+(\kappa-p) \mathcal{D}_{\jmath}\right)(\kappa-p) !}{\Omega \prod_{\jmath=1}^{\eta} \Gamma\left(\rho_{\jmath}+(\kappa-p) \mathcal{C}_{\jmath}\right)}\right]^{\ell} \frac{(\tau+p)_{\kappa-p}}{(\kappa-p) !} \mu_{\kappa} z^{\kappa}
$$

and

$$
\mathcal{J}_{p, \ell}^{\eta, \delta}\left[\sigma_{\jmath} ; \rho_{\jmath}\right] \psi(z)=\sum_{\kappa=p}^{\infty}\left[\frac{\prod_{\jmath=1}^{\delta} \Gamma\left(\sigma_{\jmath}+(\kappa-p) \mathcal{D}_{\jmath}\right)(\kappa-p) !}{\Omega \prod_{\jmath=1}^{\eta} \Gamma\left(\rho_{\jmath}+(\kappa-p) \mathcal{C}_{\jmath}\right)}\right]^{\ell} \frac{(\tau+p)_{\kappa-p}}{(\kappa-p) !} \nu_{\kappa} z^{\kappa}
$$

\section{Geometric Results}

This section introduces a certain subclass of harmonic $p$-valent functions which includes the generalized Noor-type operator $\partial_{p, \ell}^{\eta, \delta}\left[\sigma_{j} ; \rho_{j}\right] \varphi(z)$ extended to harmonic functions. This subclass is denoted by $\mathcal{H}_{p}^{\beta}\left(\alpha,\left[\sigma_{j} ; \rho_{\jmath}\right]\right)$. Further, coefficient bounds, growth formula, extreme points, convolution, convex combinations and class-preserving integral operator are also investigated for harmonic functions satisfying the subclass $\mathcal{H}_{p}^{\beta}\left(\alpha,\left[\sigma_{j} ; \rho_{\jmath}\right]\right)$.

Definition 3.1. A function $\varphi \in \mathcal{S}_{\mathcal{H}}$ is said to be in subclass $\mathcal{H}_{p}^{\beta}\left(\alpha,\left[\sigma_{j} ; \rho_{\jmath}\right]\right)$ if it satisfies the following inequality:

$$
\operatorname{Re}\left\{(1-\alpha) \frac{\partial_{p, \ell}^{\eta, \delta}\left[\sigma_{j} ; \rho_{\jmath}\right] \varphi(z)}{z^{p}}+\alpha \frac{\left[\partial_{p, \ell}^{\eta, \delta}\left[\sigma_{j} ; \rho_{\jmath}\right] \varphi(z)\right]^{\prime}}{p z^{p-1}}\right\} \geq \frac{\beta}{p}
$$


where $\mathcal{J}_{p, \ell}^{\eta, \delta}\left[\sigma_{j} ; \rho_{\jmath}\right] \varphi(z)$ is defined by $(2.7), 0 \leq \alpha \leq 1$ and $0 \leq \beta<p$.

Also, let $\mathcal{N H}_{p}^{\beta}\left(\alpha,\left[\sigma_{j} ; \rho_{\jmath}\right]\right)=\mathcal{H}_{p}^{\beta}\left(\alpha,\left[\sigma_{j} ; \rho_{\jmath}\right]\right) \cap \mathcal{N} \mathcal{S}_{\mathcal{H}(p)}$.

A sufficient coefficient condition for function belonging to the class $\mathcal{H}_{p}^{\beta}\left(\alpha,\left[\sigma_{j} ; \rho_{\jmath}\right]\right)$ is now derived.

Theorem 3.1. Let $\varphi=\phi+\bar{\psi}$ given by (1.1). Then $\varphi \in \mathcal{H}_{p}^{\beta}\left(\alpha,\left[\sigma_{j} ; \rho_{\jmath}\right]\right)$ if

$$
\begin{aligned}
& \sum_{\kappa=p+1}^{\infty}[(\kappa-p) \alpha+p]\left[\frac{\prod_{\jmath=1}^{\delta} \Gamma\left(\sigma_{\jmath}+(\kappa-p) \mathcal{D}_{\jmath}\right)(\kappa-p) !}{\Omega \prod_{\jmath=1}^{\eta} \Gamma\left(\rho_{\jmath}+(\kappa-p) \mathcal{C}_{\jmath}\right)}\right]^{\ell} \frac{(\tau+p)_{\kappa-p}}{(\kappa-p) !}\left|\mu_{\kappa}\right| \\
& +\sum_{\kappa=p}^{\infty}[(\kappa-p) \alpha+p]\left[\frac{\prod_{\jmath=1}^{\delta} \Gamma\left(\sigma_{\jmath}+(\kappa-p) \mathcal{D}_{\jmath}\right)(\kappa-p) !}{\Omega \prod_{\jmath=1}^{\eta} \Gamma\left(\rho_{\jmath}+(\kappa-p) \mathcal{C}_{\jmath}\right)}\right]^{\ell} \frac{(\tau+p)_{\kappa-p}}{(\kappa-p) !}\left|\nu_{\kappa}\right| \leq p-\beta,
\end{aligned}
$$

where $0 \leq \alpha \leq 1,0 \leq \beta<p$.

Proof. Using the fact that $\operatorname{Re}(\lambda) \geq 0$ if and only if $|1+\lambda| \geq|1-\lambda|$, it suffices to show that

$$
|p-\beta+p \theta(z)| \geq|p+\beta-p \theta(z)|,
$$

where

$$
\theta(z)=(1-\alpha) \frac{\partial_{p, \ell}^{\eta, \delta}\left[\sigma_{j} ; \rho_{\jmath}\right] \varphi(z)}{z^{p}}+\alpha \frac{\left[\partial_{p, \ell}^{\eta, \delta}\left[\sigma_{\jmath} ; \rho_{\jmath}\right] \varphi(z)\right]^{\prime}}{p z^{p-1}}
$$

Substituting for $\phi$ and $\psi$ in $\theta$, we gain

$$
\begin{aligned}
& |p-\beta+p \theta(z)| \\
\geq & 2 p-\beta-\sum_{\kappa=p+1}^{\infty}[(\kappa-p) \alpha+p]\left[\frac{\prod_{\jmath=1}^{\delta} \Gamma\left(\sigma_{\jmath}+(\kappa-p) \mathcal{D}_{\jmath}\right)(\kappa-p) !}{\Omega \prod_{\jmath=1}^{\eta} \Gamma\left(\rho_{\jmath}+(\kappa-p) \mathcal{C}_{\jmath}\right)}\right] \frac{(\tau+p)_{\kappa-p}}{(\kappa-p) !}\left|\mu_{\kappa}\right||z|^{\kappa-p} \\
& -\sum_{\kappa=p}^{\infty}[(\kappa-p) \alpha+p]\left[\frac{\prod_{\jmath=1}^{\delta} \Gamma\left(\sigma_{\jmath}+(\kappa-p) \mathcal{D}_{\jmath}\right)(\kappa-p) !}{\Omega \prod_{\jmath=1}^{\eta} \Gamma\left(\rho_{\jmath}+(\kappa-p) \mathcal{C}_{\jmath}\right)}\right]^{\ell} \frac{(\tau+p)_{\kappa-p}}{(\kappa-p) !}\left|\nu_{\kappa}\right||z|^{\kappa-p}
\end{aligned}
$$

and 


$$
\begin{aligned}
& \quad|p+\beta-p \theta(z)| \\
& \leq \beta+\sum_{\kappa=p+1}^{\infty}[(\kappa-p) a+p]\left[\frac{\prod_{\jmath=1}^{\delta} \Gamma\left(\sigma_{\jmath}+(\kappa-p) \mathcal{D}_{\jmath}\right)(\kappa-p) !}{\Omega \prod_{\jmath=1}^{\eta} \Gamma\left(\rho_{\jmath}+(\kappa-p) \mathcal{C}_{\jmath}\right)}\right]^{\ell} \frac{(\tau+p)_{\kappa-p}}{(\kappa-p) !}\left|\mu_{\kappa}\right||z|^{\kappa-p} \\
& +\sum_{\kappa=p}^{\infty}[(\kappa-p) \alpha+p]\left[\frac{\prod_{\jmath=1}^{\delta} \Gamma\left(\sigma_{\jmath}+(\kappa-p) \mathcal{D}_{\jmath}\right)(\kappa-p) !}{\Omega \prod_{\jmath=1}^{\eta} \Gamma\left(\rho_{\jmath}+(\kappa-p) \mathcal{C}_{\jmath}\right)}\right]^{\ell} \frac{(\tau+p)_{\kappa-p}}{(\kappa-p) !}\left|\nu_{\kappa}\right||z|^{\kappa-p} .
\end{aligned}
$$

These inequalities (3.4) and (3.5) in conjunction with (3.2) yields

$$
\begin{aligned}
&|p-\beta+p \theta(z)| \\
& \geq|p+\beta-p \theta(z)| \\
& \geq 2\left[(p-\beta)-\sum_{\kappa=p+1}^{\infty}[(\kappa-p) \alpha+p]\left[\frac{\prod_{\jmath=1}^{\delta} \Gamma\left(\sigma_{\jmath}+(\kappa-p) \mathcal{D}_{\jmath}\right)(\kappa-p) !}{\Omega \prod_{\jmath=1}^{\eta} \Gamma\left(\rho_{\jmath}+(\kappa-p) \mathcal{C}_{\jmath}\right)}\right] \frac{(\tau+p)_{\kappa-p}}{(\kappa-p) !}\left|\mu_{\kappa}\right|\right. \\
&\left.\quad-\sum_{\kappa=p}^{\infty}[(\kappa-p) \alpha+p]\left[\frac{\prod_{\jmath=1}^{\delta} \Gamma\left(\sigma_{\jmath}+(\kappa-p) \mathcal{D}_{\jmath}\right)(\kappa-p) !}{\Omega \prod_{\jmath=1}^{\eta} \Gamma\left(\rho_{\jmath}+(\kappa-p) \mathcal{C}_{\jmath}\right)}\right] \frac{(\tau+p)_{\kappa-p}}{(\kappa-p) !}\left|\nu_{\kappa}\right|\right] \geq 0 .
\end{aligned}
$$

The harmonic function

$$
\begin{aligned}
\varphi(z)= & z^{p}+\sum_{\kappa=p+1}^{\infty}\left[\frac{\Omega \prod_{\jmath=1}^{\eta} \Gamma\left(\rho_{\jmath}+(\kappa-p) \mathcal{C}_{\jmath}\right)}{\prod_{\jmath=1}^{\delta} \Gamma\left(\sigma_{\jmath}+(\kappa-p) \mathcal{D}_{\jmath}\right)(\kappa-p) !}\right]^{\ell} \frac{(\kappa-p) !}{(\tau+p)_{\kappa-p}[(\kappa-p) \alpha+p]} x_{\kappa} z^{\kappa} \\
& +\sum_{\kappa=p}^{\infty}\left[\frac{\Omega \prod_{\jmath=1}^{\eta} \Gamma\left(\rho_{\jmath}+(\kappa-p) \mathcal{C}_{\jmath}\right)}{\prod_{\jmath=1}^{\delta} \Gamma\left(\sigma_{\jmath}+(\kappa-p) \mathcal{D}_{\jmath}\right)(\kappa-p) !}\right]^{\ell} \frac{(\kappa-p) !}{(\tau+p)_{\kappa-p}[(\kappa-p) \alpha+p]} \bar{y}_{\kappa} \bar{z}^{\kappa},
\end{aligned}
$$

where $\sum_{\kappa=p+1}^{\infty}\left|x_{\kappa}\right|+\sum_{\kappa=p}^{\infty}\left|y_{\kappa}\right|=p-\beta$ shows that the coefficient bound given by (3.2) is sharp. 
The functions of the from (3.6) are in subclass $\mathcal{H}_{p}^{\beta}(\ell, \eta, \delta)$ because in view of (3.2), we acquire

$$
\begin{aligned}
& \sum_{\kappa=p+1}^{\infty}[(\kappa-p) \alpha+p]\left[\frac{\prod_{\jmath=1}^{\delta} \Gamma\left(\sigma_{\jmath}+(\kappa-p) \mathcal{D}_{\jmath}\right)(\kappa-p) !}{\Omega \prod_{\jmath=1}^{\eta} \Gamma\left(\rho_{\jmath}+(\kappa-p) \mathcal{C}_{\jmath}\right)}\right]^{\ell} \frac{(\tau+p)_{\kappa-p}}{(\kappa-p) !}\left|\mu_{\kappa}\right| \\
+ & \sum_{\kappa=p}^{\infty}[(\kappa-p) \alpha+p]\left[\frac{\prod_{\jmath=1}^{\delta} \Gamma\left(\sigma_{\jmath}+(\kappa-p) \mathcal{D}_{\jmath}\right)(\kappa-p) !}{\Omega \prod_{\jmath=1}^{\eta} \Gamma\left(\rho_{\jmath}+(\kappa-p) \mathcal{C}_{\jmath}\right)}\right] \frac{(\tau+p)_{\kappa-p}}{(\kappa-p) !}\left|\nu_{\kappa}\right| \\
\leq & \sum_{\kappa=p+1}^{\infty}\left|x_{\kappa}\right|+\sum_{\kappa=p}^{\infty}\left|y_{\kappa}\right|=p-\beta .
\end{aligned}
$$

This completes the proof.

Now, we yield the necessary and sufficient condition for the function $\varphi=\phi+\bar{\psi}$ given by (1.3) to be in $\mathcal{N H}_{p}^{\beta}\left(\alpha,\left[\sigma_{j} ; \rho_{\jmath}\right]\right)$.

Theorem 3.2. Let $\varphi=\phi+\bar{\psi}$ be given by (1.3). Then $\varphi \in \mathcal{N} \mathcal{H}_{p}^{\beta}\left(\alpha,\left[\sigma_{j} ; \rho_{\jmath}\right]\right)$ if and only if the condition (3.2) is as follows:

$$
\begin{aligned}
& \sum_{\kappa=p+1}^{\infty}[(\kappa-p) \alpha+p]\left[\frac{\prod_{\jmath=1}^{\delta} \Gamma\left(\sigma_{\jmath}+(\kappa-p) \mathcal{D}_{\jmath}\right)(\kappa-p) !}{\Omega \prod_{\jmath=1}^{\eta} \Gamma\left(\rho_{\jmath}+(\kappa-p) \mathcal{C}_{\jmath}\right)}\right] \frac{(\tau+p)_{\kappa-p}}{(\kappa-p) !}\left|\mu_{\kappa}\right| \\
& +\sum_{\kappa=p}^{\ell}[(\kappa-p) \alpha+p]\left[\frac{\prod_{\jmath=1}^{\delta} \Gamma\left(\sigma_{\jmath}+(\kappa-p) \mathcal{D}_{\jmath}\right)(\kappa-p) !}{\Omega \prod_{\jmath=1}^{\eta} \Gamma\left(\rho_{\jmath}+(\kappa-p) \mathcal{C}_{\jmath}\right)}\right] \frac{(\tau+p)_{\kappa-p}}{(\kappa-p) !}\left|\nu_{\kappa}\right| \leq p-\beta,
\end{aligned}
$$

where $0 \leq \alpha \leq 1,0 \leq \beta<p$.

Proof. In view of Theorem 3.1 and $\varphi \in \mathcal{N H}_{p}^{\beta}\left(\alpha,\left[\sigma_{j} ; \rho_{\jmath}\right]\right) \subset \mathcal{H}_{p}^{\beta}\left(\alpha,\left[\sigma_{j} ; \rho_{\jmath}\right]\right)$, we only need to prove the "only if" part of this theorem. Assume that $\varphi \in \mathcal{N H}_{p}^{\beta}\left(\alpha,\left[\sigma_{j} ; \rho_{\jmath}\right]\right)$, then by virtue of (3.1), we get

$$
\operatorname{Re}\left\{(p-\beta)-\sum_{\kappa=p+1}^{\infty}[(\kappa-p) \alpha+p]\left[\frac{\prod_{\jmath=1}^{\delta} \Gamma\left(\sigma_{\jmath}+(\kappa-p) \mathcal{D}_{\jmath}\right)(\kappa-p) !}{\Omega \prod_{\jmath=1}^{\eta} \Gamma\left(\rho_{\jmath}+(\kappa-p) \mathcal{C}_{\jmath}\right)}\right]^{\ell} \frac{(\tau+p)_{\kappa-p}}{(\kappa-p) !}\left|\mu_{\kappa}\right|\right.
$$




$$
\left.\times z^{\kappa-p}-\sum_{\kappa=p}^{\infty}[(\kappa-p) \alpha+p]\left[\frac{\prod_{\jmath=1}^{\delta} \Gamma\left(\sigma_{\jmath}+(\kappa-p) \mathcal{D}_{\jmath}\right)(\kappa-p) !}{\Omega \prod_{\jmath=1}^{\eta} \Gamma\left(\rho_{\jmath}+(\kappa-p) \mathcal{C}_{\jmath}\right)}\right]^{\ell} \frac{(\tau+p)_{\kappa-p}}{(\kappa-p) !}\left|\nu_{\kappa}\right| \bar{z}^{\kappa-p}\right\}
$$
$\geq 0$

This inequality (3.7) must hold for all values of $z$ in $\mathbb{D}$. Upon choosing the values of $z$ on the positive real axis, where $0<|z|=r<1$, (3.7) reduces to

$$
\begin{aligned}
& (p-\beta)-\sum_{\kappa=p+1}^{\infty}[(\kappa-p) \alpha+p]\left[\frac{\prod_{\jmath=1}^{\delta} \Gamma\left(\sigma_{\jmath}+(\kappa-p) \mathcal{D}_{\jmath}\right)(\kappa-p) !}{\Omega \prod_{\jmath=1}^{\eta} \Gamma\left(\rho_{\jmath}+(\kappa-p) \mathcal{C}_{\jmath}\right)}\right]^{\ell} \frac{(\tau+p)_{\kappa-p}}{(\kappa-p) !}\left|\mu_{\kappa}\right| r^{\kappa-p} \\
& -\sum_{\kappa=p}^{\infty}[(\kappa-p) \alpha+p]\left[\frac{\prod_{\jmath=1}^{\delta} \Gamma\left(\sigma_{\jmath}+(\kappa-p) \mathcal{D}_{\jmath}\right)(\kappa-p) !}{\Omega \prod_{\jmath=1}^{\eta} \Gamma\left(\rho_{\jmath}+(\kappa-p) \mathcal{C}_{\jmath}\right)}\right]^{\ell} \frac{(\tau+p)_{\kappa-p}}{(\kappa-p) !}\left|\nu_{\kappa}\right| r^{p-k} \geq 0 .
\end{aligned}
$$

Letting $r \rightarrow-1$ through real values, it follows that

$$
\begin{aligned}
& (p-\beta)-\sum_{\kappa=p+1}^{\infty}[(\kappa-p) \alpha+p]\left[\frac{\prod_{\jmath=1}^{\delta} \Gamma\left(\sigma_{\jmath}+(\kappa-p) \mathcal{D}_{\jmath}\right)(\kappa-p) !}{\Omega \prod_{\jmath=1}^{\eta} \Gamma\left(\rho_{\jmath}+(\kappa-p) \mathcal{C}_{\jmath}\right)}\right]^{\ell} \frac{(\tau+p)_{\kappa-p}}{(\kappa-p) !}\left|\mu_{\kappa}\right| \\
& -\sum_{\kappa=p}^{\infty}[(\kappa-p) \alpha+p]\left[\frac{\prod_{\jmath=1}^{\delta} \Gamma\left(\sigma_{\jmath}+(\kappa-p) \mathcal{D}_{\jmath}\right)(\kappa-p) !}{\Omega \prod_{\jmath=1}^{\eta} \Gamma\left(\rho_{\jmath}+(\kappa-p) \mathcal{C}_{\jmath}\right)}\right] \frac{(\tau+p)_{\kappa-p}}{(\kappa-p) !}\left|\nu_{\kappa}\right| \geq 0 .
\end{aligned}
$$

Thus, (3.8) yields (3.2). This completes the proof.

The following theorem considers the growth bounds for the function $\varphi$ that belongs to $\mathcal{N} \mathcal{H}_{p}^{\beta}\left(\alpha,\left[\sigma_{j} ; \rho_{\jmath}\right]\right)$.

Theorem 3.3. Let $\varphi \in \mathcal{N} \mathcal{H}_{p}^{\beta}\left(\alpha,\left[\sigma_{j} ; \rho_{\jmath}\right]\right)$ and $r=|z|<1$. Then

$$
|\varphi(z)| \leq\left(1+\left|\nu_{p}\right|\right) r^{p}+\left[\frac{\Omega \prod_{\jmath=1}^{\eta} \Gamma\left(\rho_{\jmath}+\mathcal{C}_{\jmath}\right)}{\prod_{\jmath=1}^{\delta} \Gamma\left(\sigma_{\jmath}+\mathcal{D}_{\jmath}\right)}\right]^{\ell} \frac{\left[p\left(1-\left|\nu_{p}\right|\right)-\beta\right]}{[\alpha+p](\tau+p)_{1}} r^{p+1}
$$


and

$$
|\varphi(z)| \geq\left(1+\left|\nu_{p}\right|\right) r^{p}-\left[\frac{\Omega \prod_{\jmath=1}^{\eta} \Gamma\left(\rho_{\jmath}+\mathcal{C}_{\jmath}\right)}{\prod_{\jmath=1}^{\delta} \Gamma\left(\sigma_{\jmath}+\mathcal{D}_{\jmath}\right)}\right]^{\ell} \frac{\left[p\left(1-\left|\nu_{p}\right|\right)-\beta\right]}{[\alpha+p](\tau+p)_{1}} r^{p+1}
$$

Proof. Let $\varphi \in \mathcal{N H}_{p}^{\beta}\left(\alpha,\left[\sigma_{j} ; \rho_{\jmath}\right]\right)$. By taking the modulus value of $\varphi$ and using Theorem 3.2, we have

$$
\begin{aligned}
& |\varphi(z)| \leq\left(1+\left|\nu_{p}\right|\right) r^{p}+\sum_{\kappa=p+1}^{\infty}\left(\left|\mu_{\kappa}\right|+\left|\nu_{\kappa}\right|\right) r^{\kappa} \\
& \leq\left(1+\left|\nu_{p}\right|\right) r^{p}+r^{p+1} \sum_{\kappa=p+1}^{\infty}\left(\left|\mu_{\kappa}\right|+\left|\nu_{\kappa}\right|\right) \\
& \leq\left(1+\left|\nu_{p}\right|\right) r^{p}+\frac{r^{p+1}}{[\alpha+p](\tau+p)_{1}}\left[\frac{\Omega \prod_{\jmath=1}^{\eta} \Gamma\left(\rho_{\jmath}+\mathcal{C}_{\jmath}\right)}{\prod_{\jmath=1}^{\delta} \Gamma\left(\sigma_{\jmath}+\mathcal{D}_{\jmath}\right)}\right]^{\ell} \\
& \times\left(\sum_{\kappa=p+1}^{\infty}[\alpha+p](\tau+p)_{1}\left[\frac{\prod_{\jmath=1}^{\delta} \Gamma\left(\sigma_{\jmath}+\mathcal{D}_{\jmath}\right)}{\Omega \prod_{\jmath=1}^{\eta} \Gamma\left(\rho_{\jmath}+\mathcal{C}_{\jmath}\right)}\right]^{\ell}\left(\left|\mu_{\kappa}\right|+\left|\nu_{\kappa}\right|\right)\right) \\
& \leq\left(1+\left|\nu_{p}\right|\right) r^{p}+\frac{r^{p+1}}{[\alpha+p](\tau+p)_{1}}\left[\frac{\Omega \prod_{\jmath=1}^{\eta} \Gamma\left(\rho_{\jmath}+\mathcal{C}_{\jmath}\right)}{\prod_{\jmath=1}^{\delta} \Gamma\left(\sigma_{\jmath}+\mathcal{D}_{\jmath}\right)}\right]^{\ell}\left(\sum_{\kappa=p+1}^{\infty}[(\kappa-p) \alpha+p]\right. \\
& \left.\times\left[\frac{\prod_{\jmath=1}^{\delta} \Gamma\left(\sigma_{\jmath}+(\kappa-p) \mathcal{D}_{\jmath}\right)(\kappa-p) !}{\Omega \prod_{\jmath=1}^{\eta} \Gamma\left(\rho_{\jmath}+(\kappa-p) \mathcal{C}_{\jmath}\right)}\right]^{\ell} \frac{(\tau+p)_{\kappa-p}}{(\kappa-p) !}\left(\left|\mu_{\kappa}\right|+\left|\nu_{\kappa}\right|\right)\right) \\
& \leq\left(1+\left|\nu_{p}\right|\right) r^{p}+\left[\frac{\Omega \prod_{\jmath=1}^{\eta} \Gamma\left(\rho_{\jmath}+\mathcal{C}_{\jmath}\right)}{\prod_{\jmath=1}^{\delta} \Gamma\left(\sigma_{\jmath}+\mathcal{D}_{\jmath}\right)}\right]^{\ell} \frac{\left[p\left(1-\left|\nu_{p}\right|\right)-\beta\right]}{[\alpha+p](\tau+p)_{1}} r^{p+1} .
\end{aligned}
$$

Also,

$$
\begin{aligned}
|\varphi(z)| & \geq\left(1+\left|\nu_{p}\right|\right) r^{p}-\sum_{\kappa=p+1}^{\infty}\left(\left|\mu_{\kappa}\right|+\left|\nu_{\kappa}\right|\right) r^{\kappa} \\
& \geq\left(1+\left|\nu_{p}\right|\right) r^{p}-\sum_{\kappa=p+1}^{\infty}\left(\left|\mu_{\kappa}\right|+\left|\nu_{\kappa}\right|\right) r^{p+1}
\end{aligned}
$$




$$
\begin{aligned}
& \geq\left(1+\left|\nu_{p}\right|\right) r^{p}-\frac{r^{p+1}}{[\alpha+p](\tau+p)_{1}}\left[\frac{\Omega \prod_{\jmath=1}^{\eta} \Gamma\left(\rho_{\jmath}+\mathcal{C}_{\jmath}\right)}{\prod_{\jmath=1}^{\delta} \Gamma\left(\sigma_{\jmath}+\mathcal{D}_{\jmath}\right)}\right]^{\ell}\left(\sum_{\kappa=p+1}^{\infty}[\alpha+p](\tau+p)_{1}\right. \\
& \left.\times\left[\frac{\prod_{\jmath=1}^{\delta} \Gamma\left(\sigma_{\jmath}+\mathcal{D}_{\jmath}\right)}{\Omega \prod_{\jmath=1}^{\eta} \Gamma\left(\rho_{\jmath}+\mathcal{C}_{\jmath}\right)}\right]^{\ell}\left(\left|\mu_{\kappa}\right|+\left|\nu_{\kappa}\right|\right)\right) \\
& \geq\left(1+\left|\nu_{p}\right|\right) r^{p}-\frac{r^{p+1}}{[\alpha+p](\tau+p)_{1}}\left[\frac{\Omega \prod_{\jmath=1}^{\eta} \Gamma\left(\rho_{\jmath}+\mathcal{C}_{\jmath}\right)}{\prod_{\jmath=1}^{\delta} \Gamma\left(\sigma_{\jmath}+\mathcal{D}_{\jmath}\right)}\right]^{\ell}\left(\sum_{\kappa=p+1}^{\infty}[(\kappa-p) \alpha+p]\right. \\
& \left.\times\left[\frac{\prod_{\jmath=1}^{\delta} \Gamma\left(\sigma_{\jmath}+(\kappa-p) \mathcal{D}_{\jmath}\right)(\kappa-p) !}{\Omega \prod_{\jmath=1}^{\eta} \Gamma\left(\rho_{\jmath}+(\kappa-p) \mathcal{C}_{\jmath}\right)}\right]^{\ell} \frac{(\tau+p)_{\kappa-p}}{(\kappa-p) !}\left(\left|\mu_{\kappa}\right|+\left|\nu_{\kappa}\right|\right)\right) \\
& \geq\left(1+\left|\nu_{p}\right|\right) r^{p}-\left[\frac{\Omega \prod_{\jmath=1}^{\eta} \Gamma\left(\rho_{\jmath}+\mathcal{C}_{\jmath}\right)}{\prod_{\jmath=1}^{\delta} \Gamma\left(\sigma_{\jmath}+\mathcal{D}_{\jmath}\right)}\right]^{\ell} \frac{\left[p\left(1-\left|\nu_{p}\right|\right)-\beta\right]}{[\alpha+p](\tau+p)_{1}} r^{p+1} .
\end{aligned}
$$

This completes the proof of Theorem 3.3.

The next theorem determines the extreme points of convex hulls of $\mathcal{N H}_{p}^{\beta}\left(\alpha,\left[\sigma_{j} ; \rho_{j}\right]\right)$ denoted by $\overline{\mathrm{co}} \mathcal{N} \mathcal{H}_{p}^{\beta}\left(\alpha,\left[\sigma_{j} ; \rho_{\jmath}\right]\right)$.

Theorem 3.4. A function $\varphi \in \overline{\mathrm{co}} \mathcal{N} \mathcal{H}_{p}^{\beta}\left(\alpha,\left[\sigma_{j} ; \rho_{\jmath}\right]\right)$ if and only if

$$
\varphi(z)=\sum_{\kappa=p}^{\infty}\left(X_{\kappa} h_{\kappa}(z)+Y_{\kappa} g_{\kappa}(z)\right)
$$

where

$$
\begin{aligned}
h_{p}(z) & =z^{p}, \\
h_{\kappa}(z) & =z^{p}-\left[\frac{\Omega \prod_{\jmath=1}^{\eta} \Gamma\left(\rho_{\jmath}+(\kappa-p) \mathcal{C}_{\jmath}\right)}{\prod_{\jmath=1}^{\delta} \Gamma\left(\sigma_{\jmath}+(\kappa-p) \mathcal{D}_{\jmath}\right)(\kappa-p) !}\right]^{\ell} \frac{(\kappa-p) !(p-\beta)}{[(\kappa-p) \alpha+p](\tau+p)_{\kappa-p}} z^{\kappa}, \\
\kappa & =p+1, p+2, \ldots,
\end{aligned}
$$




$$
\begin{aligned}
g_{\kappa}(z) & =z^{p}-\left[\frac{\Omega \prod_{\jmath=1}^{\eta} \Gamma\left(\rho_{\jmath}+(\kappa-p) \mathcal{C}_{\jmath}\right)}{\prod_{\jmath=1}^{\delta} \Gamma\left(\sigma_{\jmath}+(\kappa-p) \mathcal{D}_{\jmath}\right)(\kappa-p) !}\right]^{\ell} \frac{(\kappa-p) !(p-\beta)}{[(\kappa-p) \alpha+p](\tau+p)_{\kappa-p}} \bar{z}^{\kappa}, \\
\kappa & =p, p+1, \ldots, \\
\sum_{\kappa=p}^{\infty}\left(X_{\kappa}+Y_{\kappa}\right) & =1, X_{\kappa} \geq 0 \text { and } Y_{\kappa} \geq 0 .
\end{aligned}
$$

Proof. For a function $\varphi$ of the form (3.9), we acquire

$$
\begin{aligned}
& \varphi(z)=\sum_{\kappa=p}^{\infty}\left(X_{\kappa} h_{\kappa}(z)+Y_{\kappa} g_{\kappa}(z)\right) \\
& =X_{p} h_{p}+\sum_{\kappa=p+1}^{\infty} X_{\kappa} h_{\kappa}(z)+\sum_{\kappa=p}^{\infty} Y_{\kappa} g_{\kappa}(z) \\
& =X_{p} z^{p}+\sum_{\kappa=p+1}^{\infty} X_{\kappa} z^{p} \\
& -\sum_{\kappa=p+1}^{\infty}\left[\frac{\Omega \prod_{\jmath=1}^{\eta} \Gamma\left(\rho_{\jmath}+(\kappa-p) \mathcal{C}_{\jmath}\right)}{\prod_{\jmath=1}^{\delta} \Gamma\left(\sigma_{\jmath}+(\kappa-p) \mathcal{D}_{\jmath}\right)(\kappa-p) !}\right]^{\ell} \frac{(\kappa-p) !(p-\beta)}{[(\kappa-p) \alpha+p](\tau+p)_{\kappa-p}} X_{\kappa} z^{\kappa} \\
& +\sum_{\kappa=p}^{\infty} Y_{\kappa} z^{p}-\sum_{\kappa=p}^{\infty}\left[\frac{\Omega \prod_{\jmath=1}^{\eta} \Gamma\left(\rho_{\jmath}+(\kappa-p) \mathcal{C}_{\jmath}\right)}{\prod_{\jmath=1}^{\delta} \Gamma\left(\sigma_{\jmath}+(\kappa-p) \mathcal{D}_{\jmath}\right)(\kappa-p) !}\right]^{\ell} \\
& \times \frac{(\kappa-p) !(p-\beta)}{[(\kappa-p) \alpha+p](\tau+p)_{\kappa-p}} Y_{\kappa} \bar{z}^{\kappa} \\
& =\sum_{\kappa=p}^{\infty}\left(X_{\kappa}+Y_{\kappa}\right) z^{p} \\
& -\sum_{\kappa=p+1}^{\infty}\left[\frac{\Omega \prod_{\jmath=1}^{\eta} \Gamma\left(\rho_{\jmath}+(\kappa-p) \mathcal{C}_{\jmath}\right)}{\prod_{\jmath=1}^{\delta} \Gamma\left(\sigma_{\jmath}+(\kappa-p) \mathcal{D}_{\jmath}\right)(\kappa-p) !}\right]^{\ell} \frac{(\kappa-p) !(p-\beta)}{[(\kappa-p) \alpha+p](\tau+p)_{\kappa-p}} X_{\kappa} z^{\kappa} \\
& -\sum_{\kappa=p}^{\infty}\left[\frac{\Omega \prod_{\jmath=1}^{\eta} \Gamma\left(\rho_{\jmath}+(\kappa-p) \mathcal{C}_{\jmath}\right)}{\prod_{\jmath=1}^{\delta} \Gamma\left(\sigma_{\jmath}+(\kappa-p) \mathcal{D}_{\jmath}\right)(\kappa-p) !}\right]^{\ell} \frac{(\kappa-p) !(p-\beta)}{[(\kappa-p) \alpha+p](\tau+p)_{\kappa-p}} Y_{\kappa} \bar{z}^{\kappa}
\end{aligned}
$$




$$
\begin{gathered}
=z^{p}-\sum_{\kappa=p+1}^{\infty}\left[\frac{\Omega \prod_{\jmath=1}^{\eta} \Gamma\left(\rho_{\jmath}+(\kappa-p) \mathcal{C}_{\jmath}\right)}{\prod_{\jmath=1}^{\delta} \Gamma\left(\sigma_{\jmath}+(\kappa-p) \mathcal{D}_{\jmath}\right)(\kappa-p) !}\right]^{\ell} \frac{(\kappa-p) !(p-\beta)}{[(\kappa-p) \alpha+p](\tau+p)_{\kappa-p}} X_{\kappa} z^{\kappa} \\
-\sum_{\kappa=p}^{\infty}\left[\frac{\Omega \prod_{\jmath=1}^{\eta} \Gamma\left(\rho_{\jmath}+(\kappa-p) \mathcal{C}_{\jmath}\right)}{\prod_{\jmath=1}^{\delta} \Gamma\left(\sigma_{\jmath}+(\kappa-p) \mathcal{D}_{\jmath}\right)(\kappa-p) !}\right]^{\ell} \frac{(\kappa-p) !(p-\beta)}{[(\kappa-p) \alpha+p](\tau+p)_{\kappa-p}} Y_{\kappa} \bar{z}^{\kappa} .
\end{gathered}
$$

Therefore, in view of Theorem 3.2, we gain

$$
\left.\begin{array}{l}
\sum_{k=p+1}^{\infty}[(\kappa-p) \alpha+p]\left[\frac{\prod_{\jmath=1}^{\delta} \Gamma\left(\sigma_{\jmath}+(\kappa-p) \mathcal{D}_{\jmath}\right)(\kappa-p) !}{\Omega \prod_{\jmath=1}^{\eta} \Gamma\left(\rho_{\jmath}+(\kappa-p) \mathcal{C}_{\jmath}\right)}\right] \frac{(\tau+p)_{\kappa-p}}{(\kappa-p) !} \\
{\left[\left[\frac{\Omega \prod_{\jmath=1}^{\eta} \Gamma\left(\rho_{\jmath}+(\kappa-p) \mathcal{C}_{\jmath}\right)}{\prod_{\jmath=1}^{\delta} \Gamma\left(\sigma_{\jmath}+(\kappa-p) \mathcal{D}_{\jmath}\right)(\kappa-p) !}\right]^{\ell} \frac{(\kappa-p) !(p-\beta)}{[(\kappa-p) \alpha+p](\tau+p)_{\kappa-p}} X_{\kappa}\right]} \\
+\sum_{k=p}^{\infty}[(\kappa-p) \alpha+p]\left[\frac{\prod_{\jmath=1}^{\delta} \Gamma\left(\sigma_{\jmath}+(\kappa-p) \mathcal{D}_{\jmath}\right)(\kappa-p) !}{\Omega \prod_{\jmath=1}^{\eta} \Gamma\left(\rho_{\jmath}+(\kappa-p) \mathcal{C}_{\jmath}\right)}\right]^{\ell} \frac{(\tau+p)_{\kappa-p}}{(\kappa-p) !} \\
{\left[\left[\prod_{\jmath=1}^{\ell} \Gamma\left(\sigma_{\jmath}+(\kappa-p) \mathcal{D}_{\jmath}\right)(\kappa-p) !\right.\right.} \\
\leq
\end{array}\right]
$$

Therefore, $\varphi \in \overline{\mathrm{co}} \mathcal{N} \mathcal{H}_{p}^{\beta}\left(\alpha,\left[\sigma_{j} ; \rho_{\jmath}\right]\right)$.

Conversely, suppose that $\varphi \in \overline{\mathrm{co}} \mathcal{N} \mathcal{H}_{p}^{\beta}\left(\alpha,\left[\sigma_{j} ; \rho_{\jmath}\right]\right)$. Set

$$
\begin{gathered}
X_{\kappa}=((\kappa-p) \alpha+p)\left[\frac{\prod_{\jmath=1}^{\delta} \Gamma\left(\sigma_{\jmath}+(\kappa-p) \mathcal{D}_{\jmath}\right)(\kappa-p) !}{\Omega \prod_{\jmath=1}^{\eta} \Gamma\left(\rho_{\jmath}+(\kappa-p) \mathcal{C}_{\jmath}\right)}\right]^{\ell} \frac{(\tau+p)_{\kappa-p}}{(\kappa-p) !(p-\beta)}\left|\mu_{\kappa}\right|, \\
\kappa=p+1, p+2, \ldots,
\end{gathered}
$$


and

$$
\begin{aligned}
& Y_{\kappa}=[(\kappa-p) \alpha+p]\left[\frac{\prod_{\jmath=1}^{\delta} \Gamma\left(\sigma_{\jmath}+(\kappa-p) \mathcal{D}_{\jmath}\right)(\kappa-p) !}{\Omega \prod_{\jmath=1}^{\eta} \Gamma\left(\rho_{\jmath}+(\kappa-p) \mathcal{C}_{\jmath}\right)}\right]^{\ell} \frac{(\tau+p)_{\kappa-p}}{(\kappa-p) !(p-\beta)}\left|\nu_{\kappa}\right|, \\
& \kappa=p, p+1, p+2, \ldots
\end{aligned}
$$

On the basis of Theorem 3.2, we note that $0 \leq X_{\kappa} \leq 1, \kappa=p+1, p+2, \ldots$ and $0 \leq Y_{\kappa} \leq 1, \kappa=p, p+1, p+2, \ldots$ Let $X_{p}=1-\sum_{\kappa=p+1}^{\infty} X_{\kappa}+\sum_{\kappa=p}^{\infty} Y_{\kappa}$ and note that by Theorem 3.2, $X_{p} \geq 0$. Consequently, $\varphi(z)=\sum_{\kappa=p}^{\infty}\left(X_{\kappa} h_{\kappa}(z)+Y_{\kappa} g_{\kappa}(z)\right)$ is obtained as required.

Using convolution principle, we show the subclass $\mathcal{N} \mathcal{H}_{p}^{\beta}\left(\alpha,\left[\sigma_{\jmath} ; \rho_{\jmath}\right]\right)$ is closed under convolution.

Theorem 3.5. For $0 \leq \lambda \leq \beta<p$, let $\varphi \in \mathcal{N H}_{p}^{\beta}\left(\alpha,\left[\sigma_{j} ; \rho_{\jmath}\right]\right)$ and $\mathcal{F} \in \mathcal{N H}_{p}^{\lambda}\left(\alpha,\left[\sigma_{j} ; \rho_{\jmath}\right]\right)$. Then $\varphi * \mathcal{F} \in \mathcal{N H}_{p}^{\beta}\left(\alpha,\left[\sigma_{j} ; \rho_{\jmath}\right]\right) \subset \mathcal{N} \mathcal{H}_{p}^{\lambda}\left(\alpha,\left[\sigma_{j} ; \rho_{\jmath}\right]\right)$.

Proof. Utilizing definition of convolution, let the harmonic function $\varphi(z)=z^{p}-$ $\sum_{\kappa=p+1}^{\infty}\left|\mu_{\kappa}\right| z^{\kappa}-\sum_{\kappa=p}^{\infty}\left|\nu_{\kappa}\right| \bar{z}^{\kappa}$ and $\mathcal{F}(z)=z^{p}-\sum_{\kappa=p+1}^{\infty}\left|A_{\kappa}\right| z^{\kappa}-\sum_{\kappa=p}^{\infty}\left|B_{\kappa}\right| \bar{z}^{\kappa}$. Then, the convolution of $\varphi$ and $\mathcal{F}$ is

$$
(\varphi * \mathcal{F})(z)=z^{p}-\sum_{\kappa=p+1}^{\infty}\left|\mu_{\kappa} A_{\kappa}\right| z^{\kappa}-\sum_{\kappa=p}^{\infty}\left|\nu_{\kappa} B_{\kappa}\right| \bar{z}^{\kappa} .
$$

For $\mathcal{F} \in \mathcal{N H}_{p}^{\lambda}\left(\alpha,\left[\sigma_{j} ; \rho_{\jmath}\right]\right)$, by Theorem 3.2, we conclude that $\left|A_{\kappa}\right| \leq 1$ and $\left|B_{\kappa}\right| \leq 1$. Now for the convolution $\varphi * \mathcal{F}$, we gain

$$
\begin{aligned}
& \sum_{\kappa=p+1}^{\infty} \frac{[(\kappa-p) \alpha+p]}{(p-c)}\left[\frac{\prod_{\jmath=1}^{\delta} \Gamma\left(\sigma_{\jmath}+(\kappa-p) \mathcal{D}_{\jmath}\right)(\kappa-p) !}{\Omega \prod_{\jmath=1}^{\eta} \Gamma\left(\rho_{\jmath}+(\kappa-p) \mathcal{C}_{\jmath}\right)}\right] \frac{(\tau+p)_{\kappa-p}}{(\kappa-p) !}\left|\mu_{\kappa}\right|\left|A_{\kappa}\right| \\
+ & \sum_{\kappa=p}^{\infty} \frac{[(\kappa-p) \alpha+p]}{(p-c)}\left[\frac{\prod_{\jmath=1}^{\delta} \Gamma\left(\sigma_{\jmath}+(\kappa-p) \mathcal{D}_{\jmath}\right)(\kappa-p) !}{\Omega \prod_{\jmath=1}^{\eta} \Gamma\left(\rho_{\jmath}+(\kappa-p) \mathcal{C}_{\jmath}\right)}\right] \frac{(\tau+p)_{\kappa-p}}{(\kappa-p) !}\left|\nu_{\kappa}\right|\left|B_{\kappa}\right| \\
\leq & \sum_{\kappa=p+1}^{\infty} \frac{[(\kappa-p) \alpha+p]}{(p-\beta)}\left[\frac{\prod_{\jmath=1}^{\delta} \Gamma\left(\sigma_{\jmath}+(\kappa-p) \mathcal{D}_{\jmath}\right)(\kappa-p) !}{\Omega \prod_{\jmath=1}^{\eta} \Gamma\left(\rho_{\jmath}+(\kappa-p) \mathcal{C}_{\jmath}\right)}\right]^{\ell} \frac{(\tau+p)_{\kappa-p}}{(\kappa-p) !}\left|\mu_{\kappa}\right| \\
+ & \sum_{\kappa=p}^{\infty} \frac{[(\kappa-p) \alpha+p]}{(p-\beta)}\left[\frac{\prod_{\jmath=1}^{\delta} \Gamma\left(\sigma_{\jmath}+(\kappa-p) \mathcal{D}_{\jmath}\right)(\kappa-p) !}{\Omega \prod_{\jmath=1}^{\eta} \Gamma\left(\rho_{\jmath}+(\kappa-p) \mathcal{C}_{\jmath}\right)}\right]^{\ell} \frac{(\tau+p)_{\kappa-p}}{(\kappa-p) !}\left|\nu_{\kappa}\right| \leq 1,
\end{aligned}
$$


since $0 \leq \lambda \leq \beta<p$ and $\varphi \in \mathcal{N H}_{p}^{\beta}\left(\alpha,\left[\sigma_{j} ; \rho_{\jmath}\right]\right)$. Therefore, $\varphi * \mathcal{F} \in \mathcal{N H}_{p}^{\beta}\left(\alpha,\left[\sigma_{j} ; \rho_{\jmath}\right]\right) \subset$ $\mathcal{N} \mathcal{H}_{p}^{\lambda}\left(\alpha,\left[\sigma_{j} ; \rho_{\jmath}\right]\right)$.

In this theorem, we show that $\mathcal{N H}_{p}^{\beta}\left(\alpha,\left[\sigma_{j} ; \rho_{j}\right]\right)$ is closed under convex combination of its members. Let the functions $\varphi_{\imath}$ be defined, for $\imath=1,2, \ldots$, by

$$
\varphi_{\imath}(z)=z^{p}+\sum_{\kappa=p+1}^{\infty}\left|\mu_{\imath, \kappa}\right| z^{\kappa}-\sum_{\kappa=p}^{\infty}\left|\nu_{\imath, \kappa}\right| \bar{z}^{\kappa} .
$$

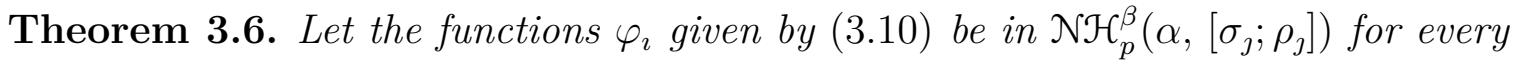
$\imath=1,2, \ldots$ Then, the function $\theta$ defined by

$$
\theta(z)=\sum_{\imath=1}^{\infty} c_{\imath} \omega_{\imath}(z), \quad 0 \leq c_{\imath}<1
$$

is also in the subclass $\mathcal{N H}_{p}^{\beta}\left(\alpha,\left[\sigma_{j} ; \rho_{\jmath}\right]\right)$, where $\sum_{\imath=1}^{\infty} c_{\imath}=1$.

Proof. According to the definition of $\theta$, we can write

$$
\theta(z)=z^{p}+\sum_{\kappa=p+1}^{\infty}\left(\sum_{\imath=1}^{\infty} c_{\imath}\left|\mu_{\imath, \kappa}\right|\right) z^{\kappa}-\sum_{\kappa=p}^{\infty}\left(\sum_{\imath=1}^{\infty} c_{\imath}\left|\nu_{\imath, \kappa}\right|\right) \bar{z}^{\kappa} .
$$

Then, by Theorem 3.2, we have

$$
\begin{aligned}
& \sum_{\kappa=p+1}^{\infty} \frac{[(\kappa-p) \alpha+p]}{(p-\beta)}\left[\frac{\prod_{\jmath=1}^{\delta} \Gamma\left(\sigma_{\jmath}+(\kappa-p) \mathcal{D}_{\jmath}\right)(\kappa-p) !}{\Omega \prod_{\jmath=1}^{\eta} \Gamma\left(\rho_{\jmath}+(\kappa-p) \mathcal{C}_{\jmath}\right)}\right] \frac{(\tau+p)_{\kappa-p}}{(\kappa-p) !}\left(\sum_{\imath=1}^{\infty} c_{\imath}\left|\mu_{\imath, \kappa}\right|\right) \\
& +\sum_{\kappa=p}^{\ell} \frac{[(\kappa-p) \alpha+p]}{(p-\beta)}\left[\frac{\prod_{\jmath=1}^{\delta} \Gamma\left(\sigma_{\jmath}+(\kappa-p) \mathcal{D}_{\jmath}\right)(\kappa-p) !}{\Omega \prod_{\jmath=1}^{\eta} \Gamma\left(\rho_{\jmath}+(\kappa-p) \mathcal{C}_{\jmath}\right)}\right] \frac{(\tau+p)_{\kappa-p}}{(\kappa-p) !}\left(\sum_{\imath=1}^{\infty} c_{\imath}\left|\nu_{\imath, \kappa}\right|\right) \\
& =\sum_{\imath=1}^{\infty} c_{\imath}\left[\sum_{\kappa=p+1}^{\infty} \frac{[(\kappa-p) \alpha+p]}{(p-\beta)}\left[\frac{\prod_{\jmath=1}^{\delta} \Gamma\left(\sigma_{\jmath}+(\kappa-p) \mathcal{D}_{\jmath}\right)(\kappa-p) !}{\Omega \prod_{\jmath=1}^{\eta} \Gamma\left(\rho_{\jmath}+(\kappa-p) \mathcal{C}_{\jmath}\right)}\right] \frac{(\tau+p)_{\kappa-p}}{(\kappa-p) !}\left|\mu_{\imath, \kappa}\right|\right. \\
& +\sum_{\kappa=p}^{\infty} \frac{[(\kappa-p) \alpha+p]}{(p-\beta)}\left[\frac{\prod_{\jmath=1}^{\delta} \Gamma\left(\sigma_{\jmath}+(\kappa-p) \mathcal{D}_{\jmath}\right)(\kappa-p) !}{\Omega \prod_{\jmath=1}^{\eta} \Gamma\left(\rho_{\jmath}+(\kappa-p) \mathcal{C}_{\jmath}\right)}\right] \\
& \leq \sum_{\imath=1}^{\infty} c_{\imath}=1 .
\end{aligned}
$$


Hence, the proof is completed.

Finally, we discuss a closure property of subclass $\mathcal{N} \mathcal{H}_{p}^{\beta}\left(\alpha,\left[\sigma_{j} ; \rho_{j}\right]\right)$ under the generalized Bernardi-Libera-Livingston integral operator $\mathcal{F}$ which is given as (see [9]):

$$
\mathcal{F}(z)=\frac{(\lambda+p)}{z^{\lambda}} \int_{0}^{z} t^{\lambda-1} \varphi(t) d t, \quad \lambda>-p .
$$

Theorem 3.7. Let $\varphi \in \mathcal{N} \mathcal{H}_{p}^{\beta}\left(\alpha,\left[\sigma_{j} ; \rho_{\jmath}\right]\right)$. Then $\mathcal{F} \in \mathcal{N H}_{p}^{\beta}\left(\alpha,\left[\sigma_{j} ; \rho_{\jmath}\right]\right)$.

Proof. Let

$$
\varphi(z)=z^{p}-\sum_{\kappa=p+1}^{\infty}\left|\mu_{\kappa}\right| z^{\kappa}-\sum_{\kappa=p}^{\infty}\left|\nu_{\kappa}\right| \bar{z}^{\kappa} .
$$

From the representation of $\mathcal{F}$, it follows that

$$
\begin{aligned}
\mathcal{F}(z) & =\frac{\lambda+p}{z^{\lambda}} \int_{0}^{z} t^{\lambda-1}\{\phi(z)+\overline{\psi(z)}\} d t \\
& =\frac{\lambda+p}{z^{\lambda}}\left\{\int_{0}^{z} t^{\lambda-1}\left(t^{p}-\sum_{\kappa=p+1}^{\infty}\left|\mu_{\kappa}\right| t^{\kappa}\right) d t-\overline{\int_{0}^{z} t^{\lambda-1}\left(\sum_{\kappa=p}^{\infty}\left|\nu_{\kappa}\right| t^{\kappa}\right)} d t\right\} \\
& =z^{p}-\sum_{\kappa=p+1}^{\infty} A_{\kappa} z^{\kappa}-\sum_{\kappa=p}^{\infty} B_{\kappa} \bar{z}^{\kappa}
\end{aligned}
$$

where

$$
A_{\kappa}=\left(\frac{\lambda+p}{\lambda+\kappa}\right)\left|\mu_{\kappa}\right| \quad \text { and } \quad B_{\kappa}=\left(\frac{\lambda+p}{\lambda+\kappa}\right)\left|\nu_{\kappa}\right| .
$$

Therefore, since $\varphi \in \mathcal{N H}_{p}^{\beta}\left(\alpha,\left[\sigma_{j} ; \rho_{\jmath}\right]\right)$,

$$
\begin{aligned}
& \sum_{\kappa=p+1}^{\infty}[(\kappa-p) \alpha+p]\left[\frac{\prod_{\jmath=1}^{\delta} \Gamma\left(\sigma_{\jmath}+(\kappa-p) \mathcal{D}_{\jmath}\right)(\kappa-p) !}{\Omega \prod_{\jmath=1}^{\eta} \Gamma\left(\rho_{\jmath}+(\kappa-p) \mathcal{C}_{\jmath}\right)}\right] \frac{(\tau+p)_{\kappa-p}}{(\kappa-p) !}\left(\frac{\lambda+p}{\lambda+\kappa}\right)\left|\mu_{\kappa}\right| \\
+ & \sum_{\kappa=p}^{\infty}[(\kappa-p) \alpha+p]\left[\frac{\prod_{\jmath=1}^{\delta} \Gamma\left(\sigma_{\jmath}+(\kappa-p) \mathcal{D}_{\jmath}\right)(\kappa-p) !}{\Omega \prod_{\jmath=1}^{\eta} \Gamma\left(\rho_{\jmath}+(\kappa-p) \mathcal{C}_{\jmath}\right)}\right]^{\ell} \frac{(\tau+p)_{\kappa-p}}{(\kappa-p) !}\left(\frac{\lambda+p}{\lambda+\kappa}\right)\left|\nu_{\kappa}\right| \\
\leq & \sum_{\kappa=p+1}^{\infty}[(\kappa-p) \alpha+p]\left[\frac{\prod_{\jmath=1}^{\delta} \Gamma\left(\sigma_{\jmath}+(\kappa-p) \mathcal{D}_{\jmath}\right)(\kappa-p) !}{\Omega \prod_{\jmath=1}^{\eta} \Gamma\left(\rho_{\jmath}+(\kappa-p) \mathcal{C}_{\jmath}\right)}\right]^{\ell} \frac{(\tau+p)_{\kappa-p}}{(\kappa-p) !}\left|\mu_{\kappa}\right|
\end{aligned}
$$




$$
+\sum_{\kappa=p}^{\infty}[(\kappa-p) \alpha+p]\left[\frac{\prod_{\jmath=1}^{\delta} \Gamma\left(\sigma_{\jmath}+(\kappa-p) \mathcal{D}_{\jmath}\right)(\kappa-p) !}{\Omega \prod_{\jmath=1}^{\eta} \Gamma\left(\rho_{\jmath}+(\kappa-p) \mathcal{C}_{\jmath}\right)}\right]^{\ell} \frac{(\tau+p)_{\kappa-p}}{(\kappa-p) !}\left|\nu_{\kappa}\right| \leq p-\beta
$$

By considering Theorem 3.2, we yield $\mathcal{F}(z) \in \mathcal{N} \mathcal{H}_{p}^{\beta}\left(\alpha,\left[\sigma_{j} ; \rho_{\jmath}\right]\right)$.

\section{Conclusion}

In this paper, we have introduced a new generalized Noor-type integral operator $g_{p, \ell}^{\eta, \delta}\left[\sigma_{j} ; \rho_{\jmath}\right]$ on the class of harmonic $p$-valent functions Correlating with FWGHfunctions in the unit disc $\mathbb{D}$. A certain subclass including this new operator is studied. In addition, some outcomes are obtained by involving coefficient condition and by showing this significance condition for negative coefficient, growth bounds, extreme points, convolution property, convex linear combination and a class-preserving integral operator.

\section{REFERENCES}

[1] O. P. Ahuja and H. Silverman, Inequalities associating hypergeometric functions with planer harmonic mapping, Journal of Inequalities in Pure and Applied Mathematics 5(4) (2004), 1-21.

[2] O. P. Ahuja and J. M. Jahangiri, Multivalent harmonic starlike functions, Ann. Univ. Mariae Curie-Sklodowska Sect. A 55 (2001), 1-13.

[3] O. P. Ahuja and P. Sharma, Inclusion theorems involving Wright's generalized hypergeometric functions and harmonic univalent functions, Acta Univ. Apulensis Math. Inform. 32 (2012), $111-128$.

[4] J. W. Alexander, Functions which map the interior of the unit circle upon simple regions, Ann. Math. 17(1) (1915), 12-22.

[5] H. F. Al-Janaby and F. Ghanim, Third-order differential Sandwich type outcome involving a certain linear operator on meromorphic multivalent functions, International Journal of Pure and Applied Mathematics 118(3) (2018), 819-835.

[6] H. F. Al-Janaby, F. Ghanim and M. Darus, Third-order differential Sandwich-type result of meromorphic p-valent functions associated with a certain linear operator, Communications in Applied Analysis 22 (2018), 63-82.

[7] H. F. Al-Janaby and M. Z. Ahmad, Differential inequalities related to Sălăgean type integral operator involving extended generalized Mittag-Leffler function, J. Phys. Conf. Ser. 1132(012061) (2019), 63-82.

[8] H. F. Al-Janaby, On certain of complex harmonic functions involving a differential operator, Journal of Advanced Research in Dynamical and Control Systems 10 (2018), 27-36.

[9] S. D. Bernardi, Convex and starlike univalent functions, Trans. Amer. Math. Soc. 135 (1969), 429-446.

[10] R. Chandrashekar, G. Murugusundaramoorthy, S. K. Lee and K. G. Subramanian, A class of complex valued harmonic functions defined by Dzoik Srivastava operator, Chamchuri J. Math. $\mathbf{1}(2)$ (2009), 31-42.

[11] J. Clunie and T. Sheil-Small, Harmonic univalent functions, Ann. Acad. Sci. Fenn. Math. 9 (1984), 3-25.

[12] L. de Branges, A proof of the Bieberbach conjecture, Acta Math. 154(1-2) (1984), 137-152.

[13] E. Deniz, On the univalence of two general integral operator, Filomat 29(7) (2015), 1581-1586. 
[14] J. Dziok and H. M. Srivastava, Classes of analytic functions associated with the generalized hypergeometric function, Appl. Math. Comput. 103(1) (1999), 1-13.

[15] J. Dziok and R. K. Raina, Families of analytic functions associated with the Wright generalized hypergeometric function, Demonstr. Math. 37(3) (2004), 533-542.

[16] R. M. El-Ashwah and M. K. Aouf, New classes of p-valent harmonic functions, Bull. Math. Anal. Appl. 2(3) (2010), 53-64.

[17] R. M. El-Ashwah, M. K. Aouf and S. M. El-Deeb, On integral operator for certain classes of p-valent functions associated with generalized multiplier transformations, J. Egyptian Math. Soc. 22 (2014), 31-35.

[18] R. M. El-Ashwah and A. H. Hassan, Third-order differential subordination and superordination results by using Fox-Wright generalized hypergeometric function, Functional Analysis: Theory, Method \& Applications 2 (2016), 34-51.

[19] C. Fox, The asymptotic expansion of generalized hypergeometric functions, Proc. London Math. Soc. 27(2) (1928), 389-400.

[20] B. A. Frasin, Univalency of general integral operator defined by Schwarz functions, J. Egyptian Math. Soc. 21 (2013), 119-122.

[21] B. A. Frasin and V. Breaz, Univalence conditions of general integral operator, Mat. Vesnik 65(3) (2013), 394-402.

[22] J. Hadamard, Théorème sur les séries entières, Acta Math. 22 (1899), 55-63.

[23] S. Hussain, A. Rasheed and M. Darus, A subclass of harmonic functions related to a convolution operator, J. Funct. Spaces 2016 (2016), 1-6.

[24] R. W. Ibrahim and M. Darus, New classes of analytic functions involving generalized Noor integral operator, J. Inequal. Appl. 2008 (2008), 1-14.

[25] A. A. Kilbas, M. Saigo and J. J. Trujillo, On the generalized Wright function, Fract. Calc. Appl. Anal. 5 (2002), 437-460.

[26] R. J. Libera, Some classes of regular univalent functions, Proc. Amer. Math. Soc. 16 (1965), $755-758$.

[27] S. S. Miller, P. T. Mocanu and M. O. Reade, Bazilevic functions and generalized convexity, Rev. Roumaine Math. Pures Appl. 19 (1974), 213-224.

[28] S. S. Miller, P. T. Mocanu and M. O. Reade, Starlike integral operators, Pacific J. Math. 79 (1978), 157-168.

[29] A. O. Mostafa, Some classes of multivalent harmonic functions defined by convolution, Electron. J. Math. Anal. Appl. 2(1) (2014), 246-255.

[30] G. Murugusundaramoorthy and R. K. Raina, On a subclass of harmonic functions associated with the Wright's generalized hypergeometric functions, Hacet. J. Math. Stat. 38(2) (2009), $129-136$.

[31] K. L. Noor, On new classes of integral operators, Journal of Natural Geometry 16 (1999), 71-80.

[32] K. L. Noor, Integral operators defined by convolution with hypergeometric functions, Appl. Math. Comput. 182(2) (2006), 1872-1881.

[33] K. W. Ong, S. L. Tan and Y. E. Tu, Integral operators and univalent functions, Tamkang J. Math. 43(2) (2012), 215-221.

[34] N. N. Pascu and V. Pescar, On integral operators of Kim-Merkes and Pfaltzgraff, Stud. Univ. Babeş-Bolyai Math. 32(55) (1990), 185-192.

[35] S. Rahrovi, On a certain subclass of analytic univalent function defined by using Komatu integral operator, Stud. Univ. Babeş-Bolyai Math. 61(1) (2016), 27-36.

[36] R. K. Raina and P. Sharma, Harmonic univalent functions associated with Wright's generalized hypergeometric function, Integral Transforms Spec. Funct. 22 (2011), 561-572.

[37] S. Ruscheweyh, New criteria for univalent functions, Proc. Amer. Math. Soc. 49 (1975), 109-115.

[38] T. M. Seoudy, On a linear combination of classes of harmonic p-valent functions defined by certain modified operator, Bull. Iranian Math. Soc. 40(6) (2014), 1539-1551. 
[39] P. Sharma, Some Wgh inequalities for univalent harmonic analytic functions, Appl. Math. 1(6) (2010), 464-469.

[40] E. M. Wright, The asymptotic expansion of the generalized hypergeometric function, J. Lond. Math. Soc. 10 (1935), 286-293.

[41] E. M. Wright, The asymptotic expansion of the generalized hypergeometric function, Proc. Lond. Math. Soc. 46(2) (1940), 389-408.

[42] E. Yaşar and S. Yalçin, Properties of a subclass of multivalent harmonic functions defined by a linear operator, General Mathematics Notes 13(1) (2012), 10-20.

${ }^{1}$ Department of Mathematics,

College of Science,

UNIVERSITY OF BAGHDAD,

BAGHDAD-IRAQ

Email address: fawzihiba@yahoo.com

${ }^{2}$ Department of Mathematics,

COllege of SCIENCE,

UNIVERSITY OF SHARJAH,

Sharjah, United Arab Emirates

Email address: fgahmed@sharjah.ac.ae 